\title{
The effects of density on the growth, survival and feed conversion of
}

\section{Tenebrio molitor larvae}

\author{
D. Deruytter" (iD) and C.L. Coudron \\ Department of aquaculture and insect rearing, Inagro, Ieperseweg 87, 8800 Rumbeke-Beitem, Belgium; \\ david.deruytter@inagro.be
}

Received: 25 March 2021 / Accepted: 22 April 2021

(C) 2021 Wageningen Academic Publishers

OPEN ACCESS CC (1) SHORT COMMUNICATION

\begin{abstract}
An appropriate stocking density is beneficial to run an efficient business and improve animal welfare. In most traditional livestock this is defined as the number of animals per area. However, the larvae of the mealworm (Tenebrio molitor), one of the most important commercial insects, live both on top and inside their feed. In this study we assessed if different areal stocking densities (2D, range 0.6-10.4 larvae $\left./ \mathrm{cm}^{2}\right)$ resulted in a different growth rate and feed efficiency when the volumetric stocking density remained constant $\left(1 \mathrm{larvae} / \mathrm{cm}^{3}\right)$. In a first experiment the larvae received wheat bran once at the start, but in the second experiment wheat bran was added throughout the experiment to ensure that the larvae remained at 1 larvae $/ \mathrm{cm}^{3}$. The results indicate that, given a stable volumetric density, the areal density can vary between 0.6 and 10.4 larvae $/ \mathrm{cm}^{2}$ without extreme variation in growth, survival or feed efficiency. The lowest assessed densities did result in a slightly lower average weight and yield. A maximum yield per volume of feed was observed at 2.5 larvae $/ \mathrm{cm}^{2}$ (feed conversion ratio (FCR) of $1.76 \mathrm{dry}$ feed/live larvae). Yet, the overall highest yield was observed at 10.4 larvae $/ \mathrm{cm}^{2}$ as the increased areal density far outweighed the slight decrease in FCR. In conclusion: when mealworm larvae are provided with an equal volume of wheat bran the influence of areal density is low and could be as high as 10.4 larvae $/ \mathrm{cm}^{2}$ for optimal yield.
\end{abstract}

Keywords: Tenebrio molitor, rearing, feed conversion, density

\section{Introduction}

The stocking density (number of animals/surface area) is a key parameter in every livestock business. Low stocking densities may result in a poor efficiency of the farm whereas too high densities (overcrowding) may result in animal welfare issues or a poor growth (Gholami et al., 2020; Sørensen et al., 2000). Insect farmers are no different from other farmers in that respect. A few studies have been performed to assess the influence of stocking density on the growth of insects (Barragan-Fonseca et al., 2018; Iba et al., 1995; Weaver and McFarlane, 1990). However, for two economically important insect species (Tenebrio molitor and Hermetia illucens) there is a major difference compared to conventional livestock that is currently not considered: the larvae live both on as in their feed. Hence, in order to assess the optimal stocking density, it may be important to consider the available volume (3D), from now on volumetric density and not (only) the available surface area (2D), from now on areal density.

For mealworm larvae, at least 2 lab-scale studies have assessed the influence of larvae density on the growth of the larvae. Weaver and McFarlane (1990) found that larvae grow faster at a higher density of 20 larvae in $400 \mathrm{ml}$ feed (approximately 0.33 larvae $/ \mathrm{cm}^{2}$ ) compared to only 2 larvae in $400 \mathrm{ml}$ feed (approximately 0.03 larvae $/ \mathrm{cm}^{2}$ ). However, the larvae reared in isolation produced larger female pupae in the end. In another study, larvae were reared in densities between 0.44 larvae $/ \mathrm{cm}^{2}$ up to 3.51 larvae $/ \mathrm{cm}^{2}$ (MoralesRamos and Rojas, 2015). In that study an equal amount of feed per larvae was added every 3 weeks without a wet feed source. They observed a significant adverse effects with increasing density on growth, feed consumption or feed efficiency. 
The goal of the current study was to explicitly assess the growth of mealworm in a semi-industrial setting with a varying areal density but an identical volumetric density. The hypothesis is that: If the areal density (number of larvae $/ \mathrm{cm}^{2}$ ) is the most important parameter, clear effects, most likely negative, should be observed between the different treatments while no effects would be observed when only the volume per insect is important. In the first experiment the larvae were fed once, therefore the volume reduced over time as the larvae consumed the feed. In the second experiment feed was replenished every week to restore it to the original volume.

\section{Material and methods}

\section{Colony information}

T. molitor has been continuously bred at the Inagro Insect Research Centre since 2013 in $60 \times 40 \mathrm{~cm}$ plastic crates (inner surface area of $2,000 \mathrm{~cm}^{2}$ ) at a temperature of $27 \pm 1^{\circ} \mathrm{C}$ $\mathrm{SD}$, a relative humidity $(\mathrm{RH})$ of $60 \pm 3 \% \mathrm{SD}$ in the dark except during feeding. The $\mathrm{CO}_{2}$ concentration is monitored and kept below $1,500 \mathrm{mg} / \mathrm{kg}$. To gather enough larvae for the experiments, $16 \times 200 \mathrm{~g}$ of beetles were allowed to lay eggs for 1 week. After oviposition, the eggs and feed (Insectus, Mijten b.v. Belgium) were collected and left at $27^{\circ} \mathrm{C}$ and $60 \%$ RH. After 7 days after oviposition wet feed was provided ad libitum as agar (25 g agar/l). 4 weeks after oviposition, the crates were gently homogenised and three subsamples were taken to estimate the number and average weight of the larvae to start the experiments with (3.5 $\mathrm{mg}$ for exp. 1 and $3.6 \mathrm{mg}$ for exp. 2).

\section{Experimental setup}

In the first experiment, 7 different larval areal densities were assessed between 0.6 and 10.4 larvae $/ \mathrm{cm}^{2}$ in triplicate (total 21 crates $(60 \times 40 \mathrm{~cm})$, Table 1$)$. Each crate was filled with wheat bran as dry feed (specific mass of $0.2 \mathrm{~kg} / \mathrm{l}$, particle size $<2 \mathrm{~mm}$ ). However, the amount of feed varied depending on the larval density (between 0.25 and $4.19 \mathrm{~kg}$ ) in such a way that all larvae received $200 \mathrm{mg}$ wheat bran. This also ensured that the larval density on a volumetric basis was identical for all treatments $\left(1\right.$ larvae $\left./ \mathrm{cm}^{3}\right)$. During the experiment, the larvae were provided ad libitum with well distributed wet feed (Agar 25 g/l, Brouwland b.v, Beverlo, Belgium, Deruytter et al., 2021). The presence of wet feed was checked daily and was added or replaced when needed. No frass was removed, and no wheat bran was added. Each week the total weight and volume of the content of the crate was determined using a ICS425 (Mettler Toledo, Zaventem, Belgium) balance and a measuring cup of the appropriate size. Thereafter, the content was gently homogenised and a subsample was taken to determine the number of larvae and the average weight using a ME203T (Mettler Toledo, average 200 larvae/subsample). After 5 weeks the experiment was terminated as the larvae started to pupate (9 weeks after starting oviposition) and an estimated 95\% of the wheat bran was consumed. The latter was defined as wheat bran larger than $0.5 \mathrm{~mm}$ with particles smaller than $0.5 \mathrm{~mm}$ defined as frass. By the end of the experiment, the initial volume had decreased by $40 \pm 5 \%$.

The second experiment was near identical to the first, with the exception that the original volume of substrate was maintained and each week the frass was removed using a $0.5 \mathrm{~mm}$ sieve. Thereafter the volume of the crate content was determined and fresh wheat bran $(0.2 \mathrm{~kg} / \mathrm{l}$, particle size $<2 \mathrm{~mm}$ ) was added to the crate to restore it to the initial volume. The larvae in experiment 2 grew slower and therefore the experiment was terminated after 6 weeks.

\section{Data analysis}

The statistical analysis was performed using $\mathrm{R}$ statistical software. A linear mixed effect model was used (Lme4 package) to assess the influence of substrate depth on the growth of mealworm larvae. Mixed effect modelling was necessary due to the longitudinal nature of the data. The analysis started using the following full model (Equation 1):

$$
\log _{10}(\text { Average weight })=\mathrm{T}^{*} \mathrm{AD}+\mathrm{T}^{2}+\mathrm{T}^{3}+\mathrm{AD}^{2}
$$

With Average weight in $\mathrm{mg}, \mathrm{T}=$ time in weeks and $\mathrm{AD}=$ areal density in larvae $/ \mathrm{cm}^{2}$. A quadratic $\left(\mathrm{T}^{2}\right.$ and $\left.\mathrm{AD}^{2}\right)$ and cubic ( $\mathrm{T}^{3}$ only) function was needed to fully describe the non-linear observed response. To determine the optimal model, the full model (equation 1) was reduced via backward selection until all parameters in the model were significant $(P$-value $<0.05)$. The data of the last week of experiment 1 and last 2 weeks of experiment 2 were not used to model the growth.

The data from the last week (week 5 for experiment 1 and week 6 for experiment 2) was used to calculate the survival (estimated number of larvae at the end/ estimated number of larvae at the start), maximum average weight and total live larvae yield per litre via linear regression. The feed conversion ratio (FCR; dry feed/ live weight gained) and frass production ratio (frass/live mealworm yield) were calculated for experiment 1 . Because feed was added throughout, and until the end of, experiment 2 it was not possible to calculate an accurate FCR and frass production.

\section{Results and discussion}

In both experiments the larvae grew well. The larvae in the first experiment grew slightly better for the first 4 weeks (Figure 1A). Yet, the larvae in the second experiment did reach a higher final weight. As both experiments occurred in identical conditions with only a few days in between, we hypothesise that this growth retardation is a result of 
Table 1. The different areal densities that were assessed in the two experiments ranging between 0.6 and $10.4 \mathrm{larvae} / \mathrm{cm}^{2}$. These were identical for both experiments. (Number of mealworm; (\#mw)).

$\begin{array}{lllllll}\text { Object } & \begin{array}{l}\text { Areal-density } \\ \left(\mathbf{M W} / \mathbf{c m}^{2}\right)\end{array} & \begin{array}{l}\text { Volumetric-density } \\ \left(\mathbf{M w} / \mathbf{c m}^{\mathbf{3}}\right.\end{array} & \begin{array}{l}\text { Initial volume } \\ \mathbf{( \mathbf { l } )}\end{array} & \begin{array}{l}\text { Wheat bran } \\ \mathbf{( k g )}\end{array} & \begin{array}{l}\text { Feed height } \\ (\mathbf{c m})\end{array} & \text { \#mw } \\ 1 & 10.4 & 1 & 21 & 4.19 & 10.4 & 21,000 \\ 2 & 6.5 & 1 & 13.1 & 2.62 & 6.5 & 13,000 \\ 3 & 4.1 & 1 & 8.2 & 1.64 & 4.1 & 8,200 \\ 4 & 2.5 & 1 & 5.1 & 1.02 & 2.5 & 5,120 \\ 5 & 1.6 & 1 & 3.2 & 0.64 & 1.6 & 3,200 \\ 6 & 1 & 1 & 2 & 0.40 & 1 & 2,000 \\ 7 & 0.6 & 1 & 1.25 & 0.25 & 0.6 & 1,250\end{array}$

the increased handling of the larvae and/or the removal of the frass. The statistical analysis indicates that in both experiments the growth was affected by the number of larvae per square centimetre (areal density, Figure 1, Table 2). Yet, in contrast to what was expected, a higher areal density resulted in a slightly better growth in both experiments. A significant positive interaction between the exposure time and areal density was observed indicating a more pronounced effect near the end of the experiments $(P<0.05)$.

The analysis on the yield data (week 5 for exp. 1 and 6 for exp. 2) reveals more insight into the influence of area and volumetric density (Figure 2, Table 3). In experiment 1 , the survival significantly declined log-linearly with an increasing areal density from an estimated $99 \%$ survival at $0.6 \mathrm{larvae} / \mathrm{cm}^{2}$ to $91 \%$ at 10.4 larvae $/ \mathrm{cm}^{2}$. This effect is not observed when the feed volume remained constant (87\%, exp. 2). However, the overall variability was higher in experiment 2 with 4 observations below an estimated $75 \%$ survival. Similar to the slightly slower growth rate (Figure 1A), we hypothesise that the, on average, higher mortality is due to the increased handling of the crates in this experiment. Future experiments should assess the impact of experimental manipulation on growth and survival of the larvae.
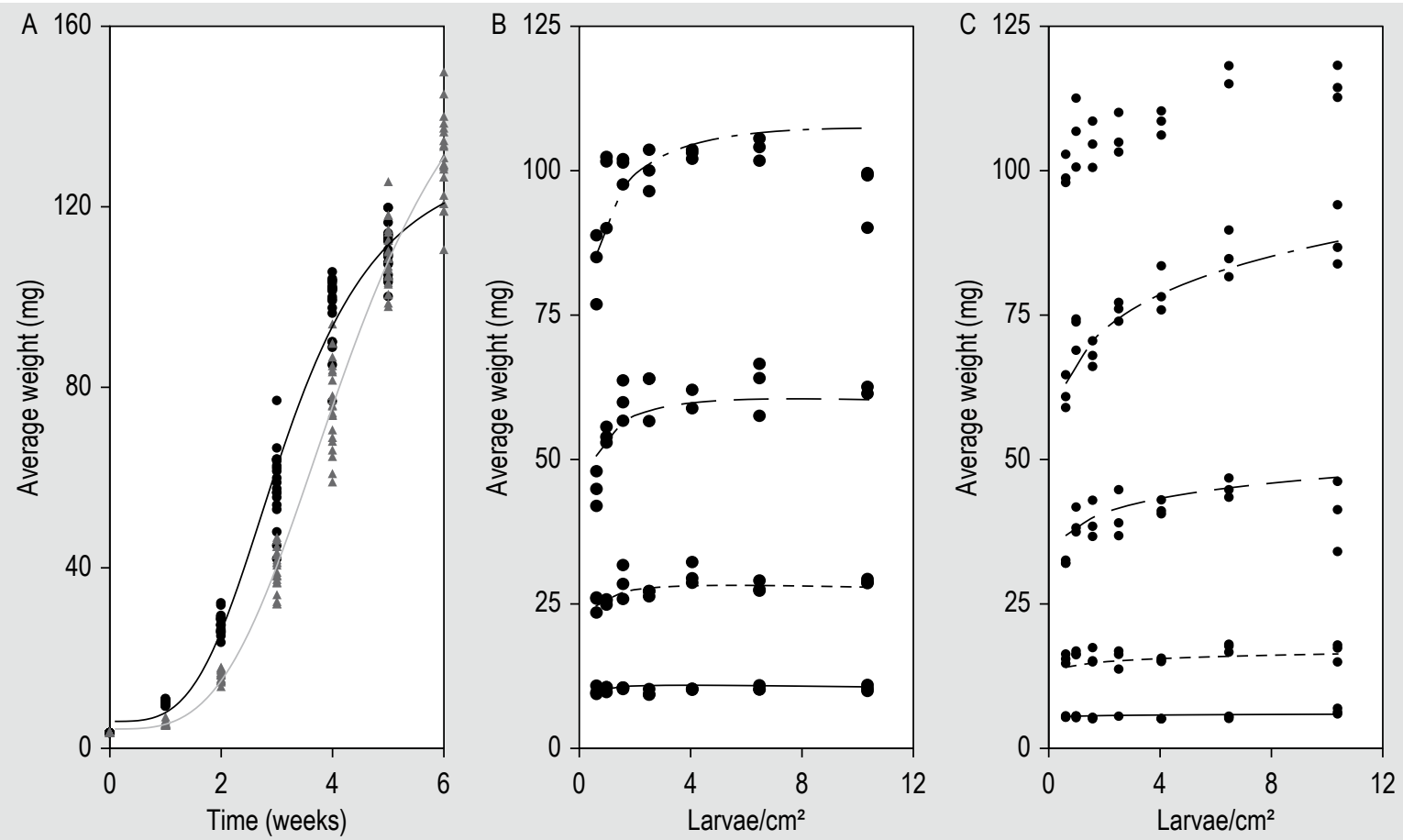

Figure 1. (A) the average growth curve for experiment 1 (black) and 2 (grey) from the start of the experiment, (B) (experiment 1) and (C) (experiment 2) the effect of areal density (larvae/ $/ \mathrm{cm}^{2}$ ) on the growth for 4 weeks (solid line = week 1 , dashed line $=$ week 2, long dashed line $=$ week 3 and dot-dashed line $=$ week 4). The average weight versus the feed volume for experiment 1 (B) and 2 (C). The individual observations (average weight of $>100$ larvae) are presented by the dots. 
Table 2. The statistical models describing the growth of the mealworms $\left(\log _{10}\right.$ weight in $\left.\mathrm{mg}\right)$ in relation to time (T [weeks]) and areal density $\left(\mathrm{AD}, \log _{10}\right.$ larvae $\left./ \mathrm{cm}^{2}\right)$ for both experiments. ${ }^{1}$

\begin{tabular}{|c|c|c|c|c|c|c|c|}
\hline Growth & Intercept & $\mathrm{T}$ & $\mathrm{T}^{2}$ & $T^{3}$ & $A D$ & $A D^{2}$ & $T \times A D$ \\
\hline Exp. 1 & $0.531(0.008)^{\star * *}$ & $0.527(0.007)$ & $-0.0423(0.0017)^{\star \star *}$ & NS & $0.0572(0.0200)^{\star *}$ & $-0.064(0.020)^{\star *}$ & $0.0189^{*}(0.0049)^{* * *}$ \\
\hline Exp. 2 & $0.560(0.009)^{\star \star *}$ & $0.0129(0.0180)$ & $0.211(0.011)^{\star \star \star}$ & $-0.0338(0.0019)^{\star \star \star}$ & $-0.00741(0.01300)$ & NS & $0.0308(0.0053)^{\star \star *}$ \\
\hline
\end{tabular}
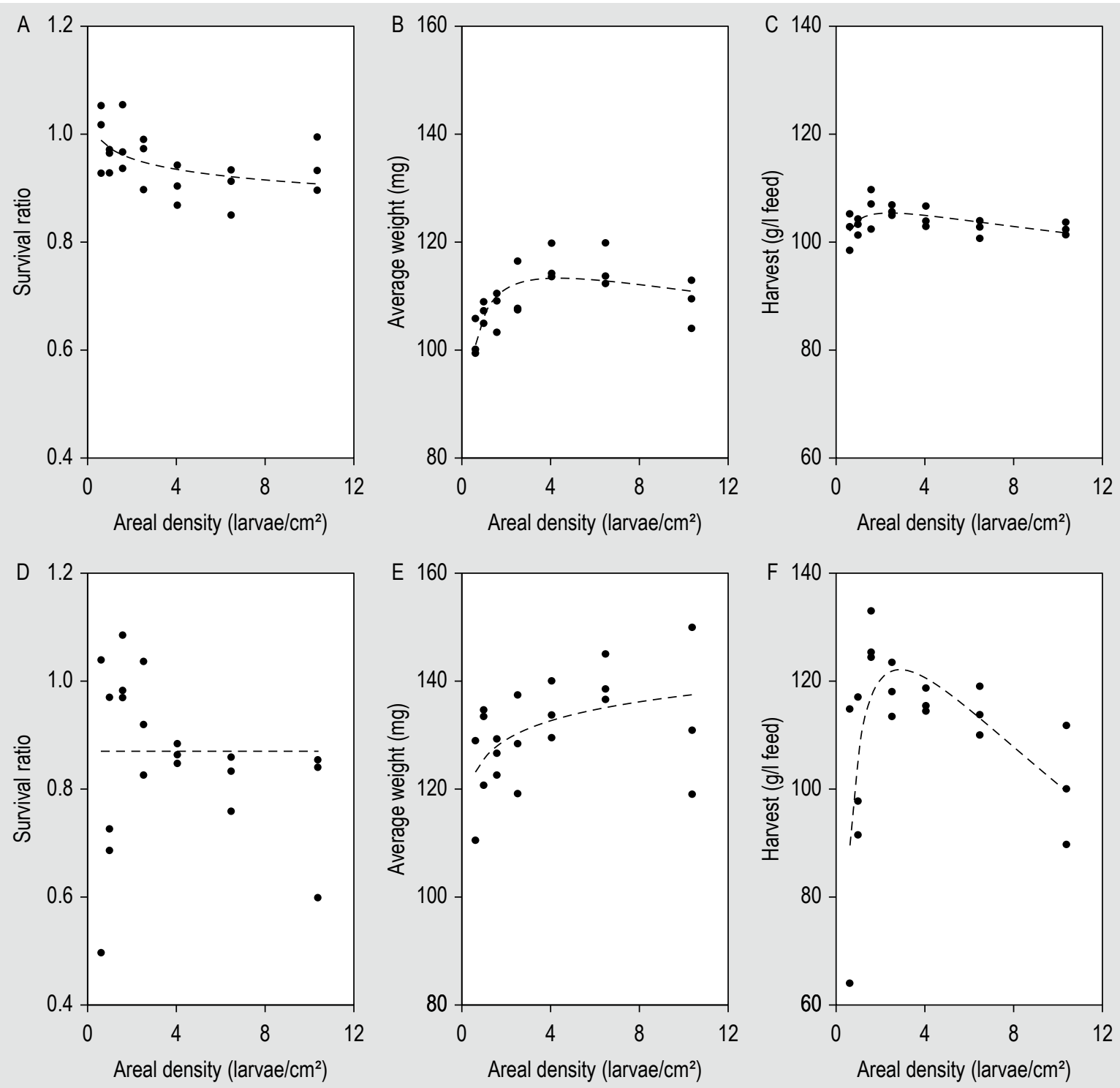

Figure 2. The survival ratio $(A, D)$, average weight of the larvae $(m g, B, E)$ and relative yield (g/l, C-F)) for experiment $1(A-C)$ and 2 (D-F) in relation to the areal density (larvae/ $\left.\mathrm{cm}^{2}\right)$. The dashed line indicates the modelled prediction (Table 2), the dots are the measured or calculated values. 
Table 3. The statistical models describing the frass production, max. weight, survival, FCR and final yield of the mealworms in relation to the areal density (AD) for both experiments. ${ }^{1}$

\begin{tabular}{|c|c|c|c|c|c|}
\hline & Parameter & Intercept & $A D$ & $A D^{2}$ & $\mathrm{R}^{2}$ \\
\hline \multirow[t]{5}{*}{ Exp. 1} & Survival & $0.974(0.015)^{\star \star *}$ & $-0.0655(0.0263)^{*}$ & NS & 0.21 \\
\hline & Max. weight & $106.36(1.24)^{\star \star *}$ & $21.8(5.4)^{* * *}$ & $-17.1(6.097)^{\star}$ & 0.50 \\
\hline & Yield $^{2}$ & $104(0.7)^{\star * \star}$ & $7.21(2.98)^{*}$ & $-9.38(3.38)^{*}$ & 0.22 \\
\hline & FCR & $1.79(0.01)^{\star \star \star}$ & $-0.137(0.0493)^{*}$ & $0.171(0.056)^{\star *}$ & 0.23 \\
\hline & Frass & $1.24(0.015)^{\star \star \star}$ & $-0.380(0.067)^{\star * *}$ & $0.500(0.076)^{\star \star *}$ & 0.68 \\
\hline \multirow[t]{3}{*}{ Exp. 2} & Max. weight & $125.7(2.82)^{\star \star \star}$ & $11.6(4.8)^{*}$ & NS & 0.20 \\
\hline & Survival & $0.87(0.05)^{\star \star \star}$ & NS & NS & 0 \\
\hline & Yield & $106.8(4.1)^{\star \star *}$ & $66.7(18.0)^{\star \star}$ & $-72.6(19.6)^{* *}$ & 0.39 \\
\hline
\end{tabular}

With $115 \mathrm{mg}$, the crates with a density of 4 larvae $/ \mathrm{cm}^{2}$ produced the heaviest larvae in experiment 1 . For experiment 2 a significant log-linear increase of the average maximum weight with increasing areal density (up to $137 \mathrm{mg}$ ) was observed. As expected, there was a significant negative correlation between the survival and maximum weight $(P<0.001$ and 0.003 for experiment 1 and 2$)$ as a lower survival results in more feed per larvae.

The amount of biomass produced per litre of feed (Figure 2) was significantly affected by the areal density for both experiments with a maximum at 2.6 larvae $/ \mathrm{cm}^{2}$ and minima at both extremes. Especially for experiment 1 , the very low difference in yield/feed volume between the assessed densities indicates that the decreased survival was offset by the increased average weight with increasing volume.

For experiment 1, a minimum FCR of 1.76 and a frass production $1.17 \mathrm{~kg} / \mathrm{kg}$ larvae was estimated at 2.5 larvae $/ \mathrm{cm}^{2}$ or a substrate height of $2.5 \mathrm{~cm}$ (Figure 3). The FCR observed in any treatment (range 1.69-1.89 $\mathrm{kg}$ feed/kg larvae) was always lower than the minimum values published in previous studies: 2.55 (Rumbos et al., 2020), 4.4 (Morales-Ramos and Rojas, 2015) and 2.62 (Van Broekhoven et al., 2015). The low FCR does indicate that the experimental conditions and assessed densities used in this study perform very good.

Although there are significant interactions between the areal density and most of the assessed parameters, our data also indicates that these effects are fairly limited when the number of animals per volume remains constant (1 larvae $/ \mathrm{cm}^{3}$ in the present study). This does indicate that the overall volume/feed per larvae is an important parameter. Based on the current data, between 2.5 and 4 larvae $/ \mathrm{cm}^{2}(2.5-4 \mathrm{~cm}$ wheat bran in a $60 \times 40$ crate and
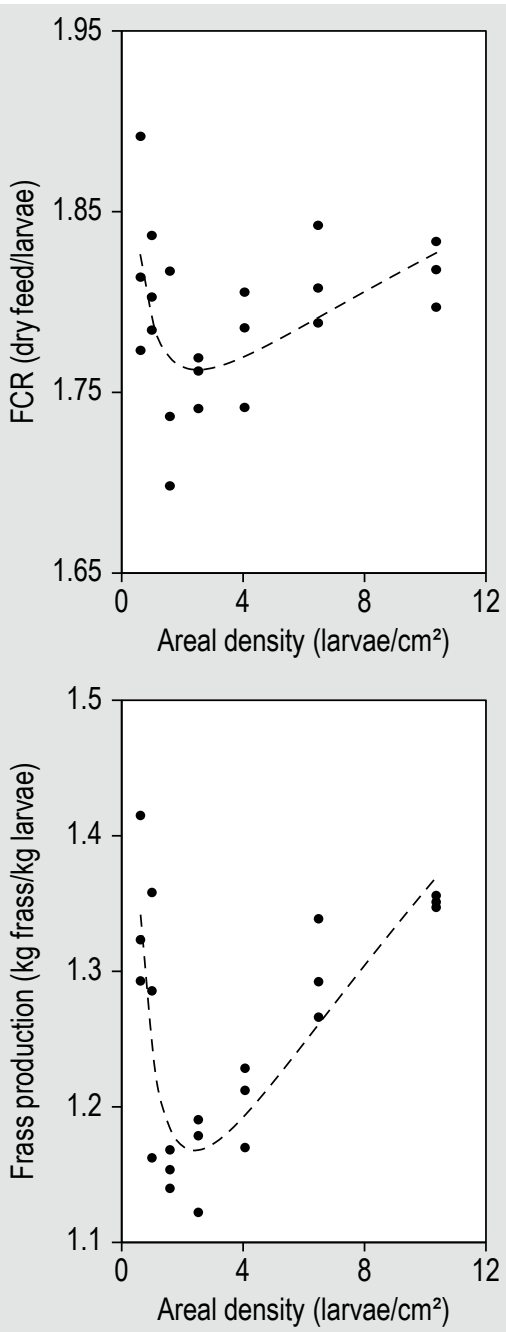

Figure 3. Top: the feed conversion ratio (FCR) at the end of experiment 1; Bottom: the frass production relative to the yield for experiment 1 in relation to the areal density (larvae $\left./ \mathrm{cm}^{2}\right)$. The dashed line indicates the modelled prediction (Table 2), the dots are the measured or calculated values. 
1 larvae $/ \mathrm{cm}^{3}$ ) is a good compromise for optimal efficiency as both the FCR and frass production are low with a high average weight and modest mortality. This is also reflected by the optimal yield/feed ratio (Figure 2). However, the sheer number of individuals that can be housed in higher density crates, despite the slightly higher mortality and reduced efficiency, results in a much higher overall yield of $2.15( \pm 0.02) \mathrm{kg} / \mathrm{crate}$ at $10.4 \mathrm{larvae} / \mathrm{cm}^{2}$ compared to 0.54 $( \pm 0.01)$ or $0.86(0.02) \mathrm{kg}$ for 2.5 to 4.1 larvae $/ \mathrm{cm}^{2}$ (Exp. 1).

The current results are consistent with Weaver et al. (1990) as they observed an enhanced growth in the first month (although female pupae were smaller in high densities by the end of the experiment). Yet, a direct comparison is difficult as the density ranges $\left(\right.$ on $\left.\mathrm{cm}^{2}\right)$ do not overlap. In another study by Morales-Ramos and Rojas (2015) comparable densities were used $\left(0.44\right.$ to $3.5 \mathrm{larvae} / \mathrm{cm}^{2}$ vs 0.6 to 10.4 larvae $/ \mathrm{cm}^{2}$ ) and they did provide all larvae an equal amount of feed, but did not specifically address the 3D matrix. In contrast to the current study, they observed a decreased growth rate and an increase in FCR with increasing areal density. It is hypothesised that this was due to increased contact with conspecifics or the increased metabolic heat production. However, there are three major differences between this study and theirs: (1) the overall size of the rearing crates: $27^{2} \mathrm{~cm}$ vs $2,000 \mathrm{~cm}^{2}$; (2) a lack of wet feed; and (3) the number of larvae per volume of feed. The latter may be the most important difference. Based on the provided data and assuming a similar feed density, MoralesRamos and Rojas (2015) used a volumetric density between 2.4 and 5.8 larvae $/ \mathrm{cm}^{3}$ compared to 1 larvae $/ \mathrm{cm}^{3}$ in the current study (increasing up to 1.7 by the end of the first experiment due to the feed being consumed). Furthermore, the density estimates (2.4-5.8) are probably conservative as the removal of the frass would decrease the available volume even further. Further research is needed to determine the optimum density per cubic centimetre instead of per square centimetre. At least one of the reasons put forward by Morales-Ramos and Rojas (2015), a reduced growth due to the increased temperatures as a result of metabolic heat production, does not seem to apply to the current study as the crate temperatures never exceeded $33^{\circ} \mathrm{C}$ which is close to the optimal temperature of $31{ }^{\circ} \mathrm{C}$ (Bjørge et al., 2018).

In conclusion, the current study is a first step towards optimising the mealworm larvae stocking density. Even when the stocking density per square centimetre varies considerably (0.6-10.4 larvae $\left./ \mathrm{cm}^{2}\right)$, the effects on growth, FCR and total yield are limited as long as the number of larvae per cubic centimetre remain equal, indicating that this is an important parameter to consider. More work is needed to assess the optimal volumetric density (larvae/ $\mathrm{cm}^{3}$ ) and how this relates to the nutritional and physical characteristics of the feed (e.g. volumetric mass density and particle size of the feed). Based on the results of the current study, 2.5-4 larvae $/ \mathrm{cm}^{2}$ (with 1 larvae $/ \mathrm{cm}^{3}$ ) results in the optimal growth but higher densities (up to 10.4 larvae $/ \mathrm{cm}^{2}$ ) will increase the overall yield with only minor adverse effects.

\section{Acknowledgments}

The authors would like to thank L. De Praetere and A. Devos for the many larvae counts. This project has received funding from the European Union's Horizon 2020 research and innovation program under grant agreement no. 861976.

\section{Conflict of interest}

The authors declare no conflict of interest.

\section{References}

Barragan-Fonseca K.B., Dicke, M. and Van Loon, J.J.A., 2018. Influence of larval density and dietary nutrient concentration on performance, body protein, and fat contents of black soldier fly larvae (Hermetia illucens). Entomologia Experimentalis et Applicata 166(9): 761-770.

Bjørge, J.D., Overgaard, J., Malte, H., Gianotten, N. and Heckmann, L.H., 2018. Role of temperature on growth and metabolic rate in the tenebrionid beetles Alphitobius diaperinus and Tenebrio molitor. Journal of Insect Physiology 107: 89-96.

Deruytter, D., Coudron, C.L. and Claeys, J., 2021. The influence of wet feed distribution on the density, growth rate and growth variability of Tenebrio molitor. Journal of Insects as Food and Feed 7(2): 141-149.

Gholami, M., Chamani, M., Seidavi, A., Sadeghi, A.A. and Aminafschar, M., 2020. Effects of stocking density and environmental conditions on performance, immunity, carcase characteristics, blood constitutes, and economical parameters of cobb 500 strain broiler chickens. Italian Journal of Animal Science 19(1): 524-535.

Iba, M., Nagao, T. and Urano, A., 1995. Effects of population density on growth, behavior and levels of biogenic amines in the cricket, Gryllus bimaculatus. Zoological Science 12(6): 695-702.

Morales-Ramos, J.A. and Rojas, M.G., 2015. Effect of larval density on food utilization efficiency of Tenebrio molitor (Coleoptera: Tenebrionidae). Journal of Economic Entomology 108(5): 2259-2267.

Rumbos, C.I., Karapanagiotidis, I.T., Mente, E., Psofakis, P. and Athanassiou, C.G., 2020. Evaluation of various commodities for the development of the yellow mealworm, Tenebrio molitor. Scientific Reports 10(1): 1-10.

Sørensen, P., Su, G. and Kestin, S.C., 2000. Effects of age and stocking density on leg weakness in broiler chickens. Poultry Science 79(6): 864-870.

Van Broekhoven, S., Oonincx, D.G.A.B., Van Huis, A. and Van Loon, J.J.A., 2015. Growth performance and feed conversion efficiency of three edible mealworm species (Coleoptera: Tenebrionidae) on diets composed of organic by-products. Journal of Insect Physiology 73: $1-10$.

Weaver, D.K. and McFarlane, J., 1990. The effect of larval density on growth and development of Tenebrio molitor. Journal of Insect Physiology 36(7): 531-536. 